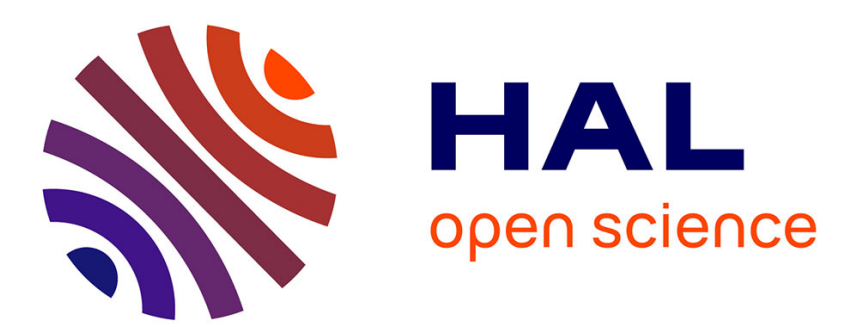

\title{
Étude générale des sondes de mesure inductive de courants. I. Description des principaux types de sondes F. Rioux-Damidau, C. Rioux
}

\section{To cite this version:}

F. Rioux-Damidau, C. Rioux. Étude générale des sondes de mesure inductive de courants. I. Description des principaux types de sondes. Revue de Physique Appliquée, 1972, 7 (4), pp.303-311. 10.1051/rphysap:0197200704030300 . jpa-00243632

\section{HAL Id: jpa-00243632 https://hal.science/jpa-00243632}

Submitted on 1 Jan 1972

HAL is a multi-disciplinary open access archive for the deposit and dissemination of scientific research documents, whether they are published or not. The documents may come from teaching and research institutions in France or abroad, or from public or private research centers.
L'archive ouverte pluridisciplinaire HAL, est destinée au dépôt et à la diffusion de documents scientifiques de niveau recherche, publiés ou non, émanant des établissements d'enseignement et de recherche français ou étrangers, des laboratoires publics ou privés. 
Classification

Physics Abstracts

05.30

\title{
ÉTUDE GÉNÉRALE DES SONDES DE MESURE INDUCTIVE DE COURANTS. I. DESCRIPTION DES PRINCIPAUX TYPES DE SONDES
}

\author{
F. RIOUX-DAMIDAU et C. RIOUX \\ Laboratoire de Physique de Plasmas, Bâtiment 214, 91, Orsay
}

(Reçu le 16 mars 1972)

\begin{abstract}
Résumé. - Nous présentons ici une étude générale des sondes de mesure inductive de courants qui comportera deux parties. Dans ce premier article, nous décrivons les principaux types de sondes : les ceintures de Rogowski, les transformateurs d'intensité et sondes à écran total, qui sont connus par ailleurs et enfin un nouveau type, les sondes à débit interne. Nous précisons leurs limitations lorsque le champ magnétique qui règne au niveau de la sonde possède la symétrie de révolution ; nous définissons alors essentiellement les coupures basses fréquences. Le prochain article abordera le problème des coupures haute fréquence et comparera les sondes entre elles.

Abstract. - A two part general study of current-measuring inductive probes is presented here. In this first part, Rogowski belts, intensity transformers and total-screening probes, which are the main classical types of probes are described, and a new « internal flow » probe is presented. Their working range and in particular the low-frequency cut-off, is evaluated for operation inside an axisymmetric magnetic field. The second part will deal with high-frequency cut-off and will present a comparative study of the different probes.
\end{abstract}

La mesure des courants intenses est un problème que l'on rencontre fréquemment à l'échelle du laboratoire comme à l'échelle industrielle et les solutions employées pour le résoudre prennent des formes variées. On atteint parfois le courant directement par une mesure aux bornes d'une résistance. D'autres fois, on préfère utiliser le champ magnétique que le courant créé à l'extérieur du circuit (sondes à effet Hall ou à effet Faraday) ou encore, la dérivée temporelle de ce champ (sondes inductives).

Les mesures effectuées aux bornes d'une résistance (par exemple Shunt de Park [1]) présentent l'avantage de répondre parfaitement aux basses fréquences, y compris au courant continu. Cependant, si leur bande passante est bonne (quelques $\mathrm{MHz}$ ) jusqu'à des courants assez intenses (quelques dizaines de $\mathrm{kA}$ ), elle diminue beaucoup lorsque les courants s'accroissent (quelques MA). D'autre part, elles ne sont pas utilisables en pratique sur des circuits portés à haute tension car dans ce cas les points de mesure ne sont pas à des potentiels voisins de zéro.

Les sondes à effet Hall ou Faraday présentent également une excellente réponse aux basses fréquences et ont de plus l'avantage d'être isolées du circuit. Malheureusement, les sondes à effet Hall coupent les hautes fréquences et l'appareillage utilisant l'effet Faraday est très important. Excepté pour les sondes inductives, tous ces procédés supposent une répartition du courant à l'intérieur du conducteur qui soit invariante au cours du temps ou tout au moins qui conserve une excellente symétrie de révolution.
Lorsque les courants à mesurer peuvent avoir une distribution spatiale variable, et être situés à des potentiels très différents de la masse, les sondes inductives paraissent seules convenir. Le but de cet exposé est d'examiner très généralement les possibilités et limites de ces sondes, dont il existe plusieurs types (les ceintures de Rogowski [2] et les transformateurs d'intensité en sont des exemples bien connus).

Dans un premier article, à la suite d'une description générale, nous examinerons les différents types de sondes concevables pour une distribution idéale du courant à mesurer présentant une complète symétrie de révolution. Cette étude nous amènera notamment à considérer un type particulier de sonde que nous appellerons «à débit interne ».

Dans un deuxième article, nous nous préoccuperons dans le détail du comportement des sondes en présence de distributions de courants dissymétriques et des limitations haute fréquence qui en résultent. Nous terminerons ce deuxième article par un diagramme comparatif général de leurs propriétés. Nous montrerons alors que le domaine complet d'utilisation de ces sondes peut être partagé entre les «transformateurs d'intensité », bien adaptés aux courants industriels (courants moyens à variations lentes) et les sondes "à débit interne » qui sont au contraire très intéressantes pour les courants intenses à variation rapide.

I. Description générale des sondes inductives (cf. Fig. 1). - Considérons un circuit conducteur $\mathrm{C}$ dans lequel circule le courant $\mathfrak{J}$ que nous désirons 


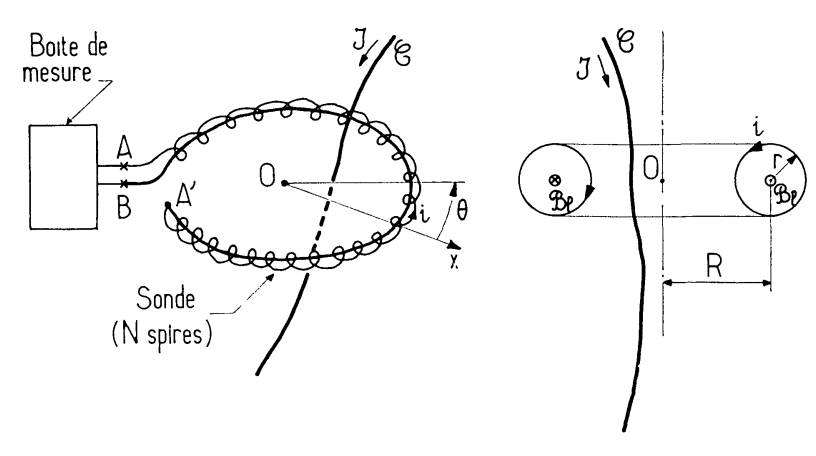

FIG. 1.

mesurer. Pour effectuer cette mesure par phénomène inductif, on dispose autour de $\mathcal{C}$ un solénoïde en forme de tore qui représente la sonde proprement dite. Aux bornes (A, B) de la sonde est installé le moyen de mesure du signal délivré.

Sur le tore, de grand rayon $R$ et de petit rayon $r$, sont enroulées $N$ spires régulièrement réparties constituées par un fil conducteur de résistivité $\rho$ et de section $s$. Un fil de retour $\mathrm{A}^{\prime} \mathrm{B}$ qui peut être de section et résistivité différentes du précédent passe par le centre des spires. Dans certains montages, le volume intérieur au tore peut être comblé par un matériau ferromagnétique (fer ou ferrite). Pour l'instant, nous ne considérerons pas ce cas.

Rappelons brièvement le calcul classique concernant ces sondes.

Lorsque le courant $J$ circule dans $\mathcal{C}$, il crée au niveau de chaque spire de la sonde une induction locale $\mathfrak{B}_{1}=\mu_{0} \mathfrak{H}_{1}$. Si l'on néglige les variations de l'induction locale $\mathcal{B}_{1}$ sur la surface d'une spire, le flux $\varphi$ crée par $\mathfrak{J}$ au travers de la sonde est :

$$
\varphi=\sum_{\text {spires }} \pi r^{2} \mathscr{B}_{1}=\pi r^{2} \sum_{\text {spires }} \mathscr{B}_{1}=\pi r^{2} \mu_{0} \sum_{\text {spires }} \mathscr{H}_{1} .
$$

De plus, si les spires régulièrement réparties sont suffisamment serrées, nous avons :

$$
\sum_{\text {spires }} \mathscr{H}_{1} \simeq \oint \frac{N}{2 \pi R} \cdot \mathscr{H}_{1} \cdot \mathrm{dl}
$$

où l'intégrale curviligne est calculée sur une courbe passant par les centres des spires. Or, d'après la relation de Maxwell-Ampère

$$
\oint \mathfrak{H}_{1} \cdot \mathrm{d} \mathrm{l} \equiv \mathfrak{J}
$$

quelle que soit la forme du conducteur $\mathcal{C}$; et par suite :

$$
\varphi=N \cdot \pi r^{2} \cdot\left(\frac{\mu_{0} J}{2 \pi R}\right)
$$

A l'aide de ce flux, on peut alors définir au niveau des spires une induction moyenne telle que :

$$
\varphi=N^{\prime} \cdot \pi r^{2} \mathscr{B}_{\mathrm{e}} \quad \text { où } \quad \mathcal{B}_{\mathrm{e}}=\frac{\mu_{0} J}{2 \pi R}
$$

$\mathscr{B}_{\mathrm{e}}$ représentera désormais le paramètre physique définissant le courant $\mathfrak{J}$ à mesurer. C'est l'induction qui existerait au niveau des spires si le conducteur $\mathrm{C}$ était confondu avec l'axe du tore.

Lorsqu'on désire extraire d'une telle sonde un signal, on constate que les conditions de fonctionnement peuvent être très différentes suivant la structure de la sonde et l'impédance de la mesure. Nous pouvons distinguer alors trois cas principaux :

1. On place entre A et B un appareil d'impédance très élevée, généralement un oscillo, capable de mesurer la tension $U_{\mathrm{AB}}$. Dans ce cas, la sonde n'est traversée par aucun courant et sa présence ne modifie pas le champ $\mathfrak{B}_{1} ;$ alors, $U_{\mathrm{AB}}=-\mathrm{d} \varphi / \mathrm{d} t \propto \mathrm{d} J / \mathrm{d} t$. Nous avons affaire à la ceinture de Rogowski (CR).

2. On court-circuite les points A et B et on utilise une sonde dont la résistance interne est très faible, de telle manière qu'elle se comporte vis-à-vis de l'induction externe $\mathcal{B}_{1}$ comme un écran total. Dans ce cas, le flux $\varphi$ au travers de la sonde est négligeable. Comme $J$ est créé par la somme du courant $\mathfrak{J}$ et du courant $i$ qui traverse la sonde, on a $: J+N i \# 0$, c'est-à-dire $i \simeq-\mathfrak{J} / N . i$ est directement proportionnel à $\mathfrak{J}$. Pour le déterminer, on remplace le court-circuit par une résistance faible aux bornes de laquelle on mesure la tension. Cet ensemble est une sonde à écran total (SET). Parfois, on est amené à utiliser un noyau ferromagnétique qui renforce les effets d'écran et l'on a alors un transformateur d'intensité (TI).

3. On court-circuite les points A et B et on utilise une sonde dont la résistance interne $\mathcal{R}$ est très élevée. Le courant $i$ est régi par l'équation $R i=-\mathrm{d} \varphi_{\mathrm{r}} / \mathrm{d} t$ où $\varphi_{\mathrm{T}}$ est encore le flux total engendré par le courant total $\mathfrak{J}+N i$. Si $\mathcal{R}$ est suffisamment grand, $i$ est petit et la contribution de ce dernier au flux $\varphi_{\mathrm{T}}$ est négligeable. L'on a alors

$$
i \simeq-\frac{1}{\mathcal{R}} \frac{\mathrm{d} \varphi}{\mathrm{d} t} \sim-\frac{1}{\mathcal{R}} \frac{\mathrm{d} J}{\mathrm{~d} t} .
$$

Pour déterminer $J$ et $\mathrm{d} J / \mathrm{d} t$, on remplace le courtcircuit par une résistance $R_{\mathrm{m}}$ et une capacité $C_{\mathrm{m}}$ dont les impédances sont faibles comparées à $\mathcal{R}$. La mesure de la tension aux bornes de $R_{\mathrm{m}}$ donne $\mathrm{d} J / \mathrm{d} t$; celle de la tension aux bornes de $C_{\mathrm{m}}$ donne $\mathrm{J}$. Nous distinguerons ces sondes sous le nom de « sondes à débit interne » (SDI).

Le but de cet article est de préciser les domaines d'utilisation de ces trois types de sondes. Pour cela, nous calculerons le signal qu'elles fournissent pour un courant sinusoïdal de pulsation $\omega$ :

$$
\mathfrak{J}=I \sin (\omega t+\varphi) .
$$

En représentation complexe, nous aurons :

$$
\bar{J}(t)=\bar{I} \mathrm{e}^{j \omega t}
$$

où $\bar{I}$ est une constante complexe.

Par convention, nous noterons $|\bar{I}|=I$, et nous 
appliquerons cette écriture à toutes les grandeurs variables.

Nous délimiterons les domaines d'utilisation, en partant des conditions d'emploi suivantes :

- Le signal de mesure reproduit $\mathfrak{J}$ ou $\mathrm{d} J / \mathrm{d} t$ à une précision au moins égale à $g$ (la classe de la sonde est alors $100 \mathrm{~g}$ ).

- Les tensions à mesurer doivent avoir une valeur compatible avec des mesures aisées, c'est-à-dire comprises entre deux valeurs extrêmes $U_{\mathrm{m}}$ et $U_{\mathrm{M}}$ facilement mesurables à l'oscilloscope. $U_{\mathrm{M}}$ est d'ailleurs tel qu'il n'y a aucun risque de claquage dans la sonde.

Pour étudier les conditions d'emploi relatives de ces sondes, nous choisirons dans cette étude les valeurs :

$$
\begin{aligned}
g & =1 \% \\
U_{\mathrm{m}} & =1 \mathrm{mV} \\
U_{\mathrm{M}} & =300 \mathrm{~V} .
\end{aligned}
$$

Les conclusions relatives que nous obtiendrons ne seront pas modifiées dans leur portée générale si l'on change ces valeurs, même de manière importante.

A la suite de ces considérations globales, nous allons maintenant étudier séparément chacun des trois types de sondes.

II. Ceintures de Rogowski. - 1. Signal. - Le signal est représenté par la tension $\mho_{\mathrm{AB}}$, laquelle est proportionnelle à $\mathrm{d} J / \mathrm{d} t$.

En effet :

$$
\mathrm{U}_{\mathrm{AB}}=-\frac{\mathrm{d} \varphi}{\mathrm{d} t}=-\pi N r^{2} \frac{\mathrm{d} \mathscr{\mathcal { H }}_{\mathrm{e}}}{\mathrm{d} t}
$$

Le module de cette tension est égal à :

$$
U_{\mathrm{AB}}=\pi N r^{2} \omega B_{\mathrm{e}} \text {. }
$$

2. Domaine D'Utilisation. - Pour que le signal soit mesurable il doit tout d'abord satisfaire aux inéquations

$$
U_{\mathrm{m}} \leqslant U_{\mathrm{AB}} \leqslant U_{\mathrm{M}}
$$

ou encore :

$$
U_{\mathrm{m}} \leqslant \pi r^{2} \omega N B_{\mathrm{e}} \leqslant U_{\mathrm{M}}
$$

D'autre part, l'utilisation aux hautes fréquences met en jeu des phénomènes capacitifs qui vont $a$ priori perturber notre description simple. Evaluons donc leur influence. En ce qui concerne sa capacité, la sonde est sensiblement équivalente à un câble coaxial dont l'âme centrale serait le fil de retour $\mathrm{A}^{\prime} \mathrm{B}$ et la tresse de masse le fil de bobinage $\mathrm{AA}^{\prime}$. Si le câble de mesure est plus long que la circonférence $2 \pi R$ de la sonde, c'est la capacité de ce dernier qui limitera la fréquence du courant mesurable; ce sera le cas général. Par contre, l'inductance du câble est totalement négligeable par rapport à celle de la sonde. Il en résulte que l'ensemble câble-sonde peut grosso modo être assimilé à un circuit constitué par une capacité ponc- tuelle $C_{0}$ située sur le câble et une inductance $L$ également ponctuelle formée par la sonde proprement dite. La pulsation propre de ce circuit est égale à $\omega_{0}=1 / \sqrt{L C_{0}}$.

Pour que la précision de la mesure soit supérieure à $g$, il est facile de montrer que $\omega$ doit satisfaire l'inéquation

$$
\omega \leqslant \sqrt{g} \omega_{0}
$$

ou encore :

$\frac{\omega N r}{\sqrt{R}} \leqslant \sqrt{2} \sqrt{g} \cdot \frac{1}{\sqrt{\mu_{0}}} \cdot \frac{1}{\sqrt{C_{0}}}=0,1 \sqrt{2} \cdot \frac{1}{\sqrt{\mu_{0}}} \cdot \frac{1}{\sqrt{C_{0}}}$ pour la valeur $g=10^{-2}$ admise ici.

La capacité d'un câble est de l'ordre de 100 pF/m tandis que la capacité d'entrée d'un oscilloscope est de $50 \mathrm{pF}$; aussi, pour préciser numériquement les limitations hautes fréquences, nous prendrons la valeur minimale $C_{0}=100 \mathrm{pF}$.

Pour étudier le comportement d'une sonde donnée en fonction de la pulsation $\omega$ et du module $I$ du courant variable $J$ (défini implicitement par l'induction moyenne $B_{\mathrm{e}}$ au niveau de la sonde), nous utiliserons d'une part le paramètre $N r \omega / \sqrt{R}$ ne dépendant pas de $B_{\mathrm{e}}$ et d'autre part le paramètre

$$
\frac{r^{2} \omega N B_{\mathrm{e}}}{\frac{N r \omega}{\sqrt{R}}}=r \sqrt{R} B_{\mathrm{e}}=\frac{\mu_{0}}{2 \pi} \cdot \frac{r}{\sqrt{R}} I
$$

qui ne dépend pas de la fréquence.

A l'aide de ces deux paramètres, les inéquations définissant le comportement de la sonde s'écrivent :

$$
\begin{aligned}
& \frac{U_{\mathrm{m}}}{\pi} \cdot \frac{1}{r \sqrt{R} B_{\mathrm{e}}} \leqslant \frac{N r \omega}{\sqrt{R}} \leqslant \frac{U_{\mathrm{M}}}{\pi} \cdot \frac{1}{r \sqrt{R} B_{\mathrm{e}}} \\
& \frac{N r \omega}{\sqrt{R}} \leqslant 0,1 \frac{\sqrt{2}}{\sqrt{\mu_{0}}} \cdot \frac{1}{\sqrt{C_{0}}} .
\end{aligned}
$$

Le diagramme de la figure 2 représente ces inéquations pour les valeurs numériques que nous avons

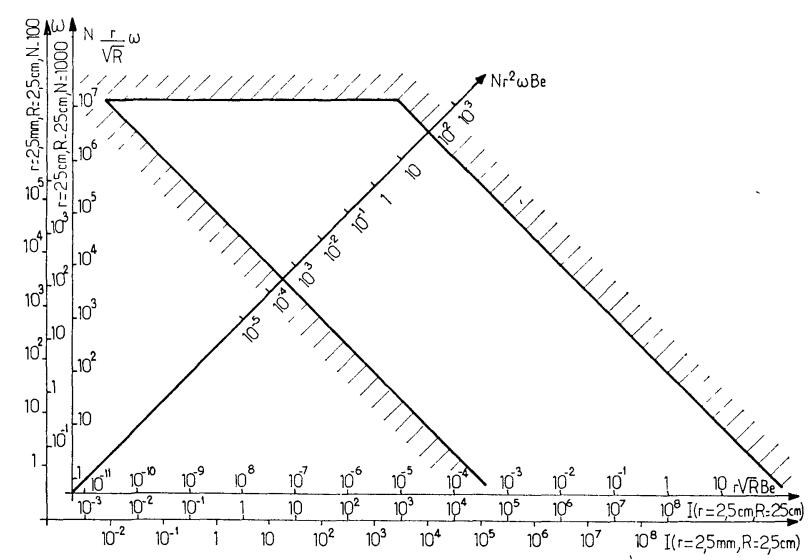

FIG. 2. 
introduites plus haut $\left(U_{\mathrm{m}}=1 \mathrm{mV}, U_{\mathrm{M}}=300 \mathrm{~V}\right.$, $\left.C_{0}=100 \mathrm{pF}\right)$. En plus des paramètres déjà cités, nous avons indiqué en abscisse la valeur de $I$ pour $r=2,5 \mathrm{~mm}$ et $R=2,5 \mathrm{~cm}$ d'une part, $r=2,5 \mathrm{~cm}$ et $R=25 \mathrm{~cm}$ d'autre part ; ces valeurs diffèrent d'ailleurs très peu puisque $r \sqrt{R} B_{\mathrm{e}}$ est proportionnel à $(r / \sqrt{R}) I$. En ordonnée, nous avons porté $\omega$ pour deux géométries différentes des sondes $(r=2,5 \mathrm{~mm}, R=2,5 \mathrm{~cm}$, $N=100$ pour la première et $r=2,5 \mathrm{~cm}, R=25 \mathrm{~cm}$ et $N=1000$ pour la seconde). Ces valeurs typiques nous permettent de nous faire une idée rapide du domaine d'utilisation des CR.

Remarquons que pour les courants inférieurs à $10^{3} \mathrm{~A}$ environ, la coupure haute fréquence est déterminée par la capacité $C_{0}$ et est indépendante de la valeur de $I$; la valeur de cette limitation dépend des paramètres géométriques mais atteint difficilement $1 \mathrm{MHz}$. Au-delà de $10^{3} \mathrm{~A}$, la limitation aux fréquences élevées dépend du courant. Plus précisément, compte tenu de (5) et de $B_{\mathrm{e}}=\mu_{0} I / 2 \pi R$, nous avons :

$$
I \omega \leqslant \frac{2 U_{\mathrm{M}}}{\mu_{0}} \cdot \frac{R}{N r^{2}} .
$$

Pour $U_{\mathrm{M}}=300 \mathrm{~V}, N=100, r=2,5 \mathrm{~mm}, R=2,5 \mathrm{~cm}$, nous avons :

$$
I \omega \leqslant 2 \times 10^{10} .
$$

Pour $U_{\mathrm{M}}=300 \mathrm{~V}, N=1000, r=2,5 \mathrm{~cm}, R=25 \mathrm{~cm}$, nous avons :

$$
I \omega \leqslant 2 \times 10^{8} .
$$

Les $\mathrm{CR}$ ne peuvent mesurer de courants élevés à variation rapide car les tensions de mesure deviendraient prohibitives.

Inversement, pour que la tension à mesurer soit suffisante, il faut que le produit $I \omega$ ne soit pas trop petit (supérieur à $7 \times 10^{4}$ pour la petite sonde et $7 \times 10^{2}$ pour la grande).

De façon générale, si l'on désire atteindre avec ces sondes principalement les basses fréquences ou tout au contraire les hautes fréquences, on est amené à choisir des géométries adaptées à chacun de ces problèmes. Les conditions limites d'emploi des sondes pour ces géométries particulières seront précisées dans le prochain article.

II. Sondes à écran total. Transformateurs d'intensité. - 1. Signal. - Nous avons déjà dit que ce type de sonde fait totalement écran au champ magnétique et que par suite elle est parcourue par un courant $i$ tel que $N i \simeq J$ ( $N$ étant le nombre de spires). Un ampèremètre placé entre $\mathrm{A}$ et $\mathrm{B}$ mesure $i$; le signal de mesure est donc directement proportionnel au courant.

2. Conditions D'Utilisation. - Le schéma de ces sondes est donné en coupe sur la figure 3.

Leur structure est globalement semblable à celle des CR. Elle en diffère essentiellement par la grosseur

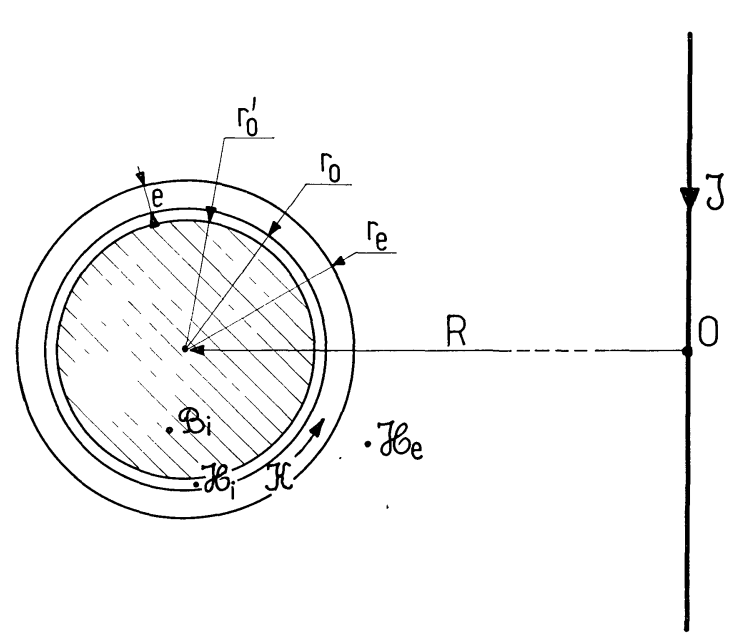

FIG. 3.

$\mathrm{du}$ fil $\mathrm{du}$ bobinage, qui est toujours en cuivre pour assurer un bon écran, et par l'emploi dans certains cas d'un noyau ferromagnétique destiné à renforcer cet effet d'écran. Nous admettrons, dans ce dernier cas, que la perméabilité relative $\mu_{\mathrm{r}}$ du noyau reste constante et égale à une valeur de l'ordre de $10^{4}$ jusqu'à la saturation. Nous avons représenté la sonde par un tore conducteur équivalent d'épaisseur régulière $e=N s / 2 \pi R$, de petit rayon intérieur $r_{0}$, de petit rayon extérieur $r_{\mathrm{e}}\left(r_{\mathrm{e}}-r_{0}=e\right)$. Nous admettrons que l'on a toujours : $e \ll r$ avec $r_{\mathrm{e}} \simeq r_{0} \simeq r$ et que $R \gg r$. Nous appellerons $\pi$ la densité de courant dans l'épaisseur continue du tore.

Pour calculer la réponse de la sonde, nous supposerons tout d'abord que le courant $\mathfrak{J}$ est porté par l'axe du tore, comme cela est indiqué sur la figure 3 . Le champ magnétique reste indépendant de la variable angulaire $\theta$ (voir Fig. 1) à la fois à l'intérieur et à l'extérieur du tore.

$\mathrm{Au}$ voisinage extérieur du tore, nous avons $\mathfrak{H}_{\mathrm{e}}=\mathfrak{J} / 2 \pi R$. Si la sonde ne fait pas totalement écran, il règne en son intérieur un champ magnétique $\mathscr{H}_{\mathrm{i}}$ et une induction $\mathcal{B}_{\mathrm{i}}=\mu_{0} \mu_{\mathrm{r}} \mathcal{H}_{\mathrm{i}}$. Les champs $\mathscr{H}_{\mathrm{e}}$ et $\mathcal{H}_{\mathrm{i}}$ sont reliés instantanément à $i$ et $\mathcal{J}$ par la relation :

$$
\frac{J-N_{i}}{J}=\frac{\mathfrak{H}_{\mathrm{i}}}{\mathfrak{H}_{\mathrm{e}}}
$$

Le rapport $\mathcal{H}_{\mathrm{i}} / \mathcal{H}_{\mathrm{e}}$ qui exprime la pénétration du champ est directement lié à la précision de la mesure.

Si nous convenons de représenter toutes les grandeurs sinusoïdales de pulsation $\omega$ en écriture complexe, la précision relative de la mesure peut s'exprimer par le rapport :

$$
\frac{H_{\mathrm{i}}}{H_{\mathrm{e}}}=\frac{I-N_{i}}{I} \leqslant g=10^{-2}
$$

Le but d'une sonde est de produire un signal de mesure pour lequel $g$ soit aussi petit que possible dans une large plage de fonctionnement. Pour cela, 
il faut résoudre conjointement un certain nombre de problèmes annexes liés principalement à :

- la tenue des efforts mécaniques engendrés par les forces magnétiques;

- l'échauffement de la sonde ;

- l'influence de l'inhomogénéité des champs et des capacités parasites.

Ce dernier problème sera traité dans un prochain article.

En admettant que les conditions de tenue mécanique sont toujours satisfaites par une construction adaptée, nous nous proposons maintenant d'examiner, d'une part, la pénétration du champ lorsque sa répartition dans la sonde est homogène et, d'autre part, l'échauffement de cette sonde.

๙) Pénétration du champ. - Elle sera d'autant plus faible que le conducteur sera meilleur: pour toutes les applications numériques, nous admettrons que la sonde est en cuivre.

$\mathrm{Au}$ travers de l'épaisseur du tore, les équations d'évolution du champ électromagnétique s'obtiennent classiquement à partir des équations de Maxwell et correspondent à une diffusion. Dans cette étude où nous cherchons essentiellement des ordres de grandeur, nous admettrons que $r_{\mathrm{e}}-r_{0}=e \ll r_{0}$. Alors, pour la grandeur complexe $\bar{k}=\bar{k}(r)$ représentant la densité de courant, cette équation s'écrit :

$$
\frac{\mathrm{d}^{2} \bar{k}}{\mathrm{~d} r^{2}}=2 j \frac{\bar{k}}{\delta^{2}},
$$

où $j^{2}=-1$ et $\delta^{2}=2 \rho / \mu_{0} \omega$ est l'épaisseur de peau $\mathrm{du}$ conducteur [3].

Pour préciser les conditions aux limites, nous lierons le flux intérieur $\varphi$ au champ électrique au voisinage du rayon $r_{0}$, et écrirons :

$$
\oint \bar{E} \mathrm{~d} l=-\frac{\overline{\mathrm{d} \varphi}}{\mathrm{d} t} .
$$

En posant $\bar{k}\left(r_{0}\right)=\bar{k}_{0}$ et $\bar{\varphi}=\bar{\varphi}(r) \mathrm{e}^{j \omega t}$, nous avons :

$$
2 \pi r_{0} \rho \bar{k}_{0}=-j \omega \bar{\varphi} \text {. }
$$

En l'absence de matériau magnétique, $\bar{\varphi}=\pi r_{0}^{2} \bar{B}_{\mathrm{i}}$. En présence d'un noyau magnétique de forte perméabilité pour lequel $r_{0}^{\prime} \# r_{0}$, nous avons encore $\bar{\varphi} \# \pi r_{0}^{2} \bar{B}_{\mathrm{i}}$. Par suite, nous obtenons dans tous les cas la condition aux limites déduite de (10):

$$
\bar{k}_{0}=-j \omega \frac{r_{0}}{2 \rho} \bar{B}_{\mathrm{i}} .
$$

Tous calculs effectués, l'expression du champ $\overline{H_{\mathrm{i}}}=\overline{B_{\mathrm{i}}} / \mu$ intérieur au tore s'écrit alors :

$$
H_{\mathrm{i}}=\frac{B_{\mathrm{e}}}{\mu_{0}} \cdot \frac{1}{1+\mu_{\mathrm{r}} \frac{r_{0}}{e} \overline{D\left(\frac{e}{\delta}\right)}}
$$

où

$$
\overline{D\left(\frac{e}{\delta}\right)}=\frac{1+j}{2} \cdot \frac{e}{\delta}\left\{\exp \left[(1+j) \frac{e}{\delta}\right]-1\right\} .
$$

Si nous désirons effectuer la mesure avec une précision au moins égale à $g$, nous devrons avoir entre les modules des champs, la relation :

$$
H_{\mathrm{i}} \leqslant g H_{\mathrm{e}}=g \frac{B_{\mathrm{e}}}{\mu_{0}}
$$

ou encore :

$$
\left|\frac{1}{1+\mu_{\mathrm{r}} \frac{r_{0}}{e} \overline{D\left(\frac{e}{\delta}\right)}}\right| \leqslant g .
$$

Comme $g \ll 1$, cette condition entraîne :

$$
\mu_{\mathrm{r}} \cdot \frac{r_{0}}{e} \overline{D\left(\frac{e}{\delta}\right)} \geqslant \frac{1}{g} \gg 1
$$

et le module du champ $\mathscr{H}_{\mathrm{i}}$ prend la forme simplifiée :

$$
H_{\mathrm{i}} \simeq \frac{1}{\mu_{\mathrm{r}} \frac{r_{0}}{e} \overline{D\left(\frac{e}{\delta}\right)}} \cdot \frac{B_{\mathrm{e}}}{\mu_{0}}
$$

En outre, lorsqu'il y a un noyau ferromagnétique, il faut veiller à ce que l'induction dans le tore garde une valeur inférieure à la valeur $B_{\mathrm{S}}$ de la saturation, c'est-à-dire : $B_{\mathrm{i}}=\mu_{0} \mu_{\mathrm{r}} H_{\mathrm{i}} \leqslant B_{\mathrm{S}}$. En remplaçant $H_{\mathrm{i}}$ par sa valeur en fonction de $B_{\mathrm{e}}$ (éq. (14)), cette relation devient :

$$
\frac{r_{0}}{e} \overline{D\left(\frac{e}{\delta}\right)} \geqslant \frac{B_{\mathrm{e}}}{B_{\mathrm{s}}} .
$$

Bien évidemment, pour $\mu_{\mathrm{r}}=1$, la dernière inéquation n'a pas de sens.

Les éq. (13) et (15) ne font intervenir que les termes

$$
\frac{r_{0}}{e} D\left(\frac{e}{\delta}\right) \text { et } \frac{B_{\mathrm{e}}}{B_{\mathrm{s}}} \text {. }
$$

Nous pouvons représenter simplement les domaines qu'elles délimitent dans un diagramme (voir Fig. 4) en coordonnées

$$
\left\{\frac{B_{\mathrm{e}}}{B_{\mathrm{s}}}, \frac{r_{0}}{e} \overline{D\left(\frac{e}{\delta}\right)}\right\}
$$

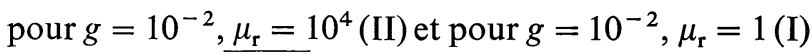
L'ordonnée $\frac{r_{0}}{e} \overline{D\left(\frac{e}{\delta}\right)}$ dépend de $\delta$, c'est-à-dire de $\omega$ pour la relation $\delta^{2}=\frac{2 \rho}{\mu_{0} \omega}$. Pour un emploi usuel, on a :

$$
\frac{r_{0}}{e} \overline{D\left(\frac{e}{\delta}\right)} \propto \omega \text {. }
$$

Nous voyons sur cette figure que le domaine d'utilisation des sondes à noyau ferromagnétique est très nettement différent de celui des sondes sans noyau. 


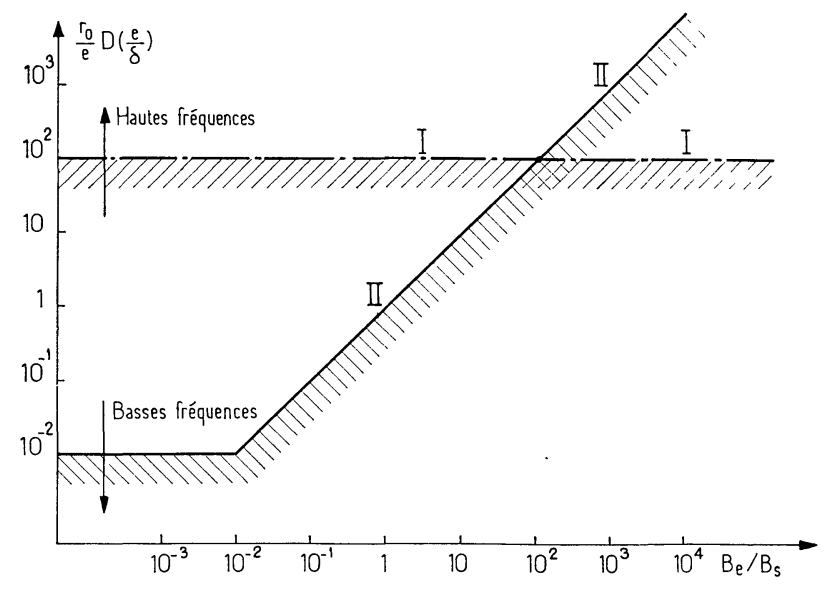

FIG. 4.

Nous sommes aussi amenés à distinguer deux types de sondes :

- Celles du premier type ne comportent pas de noyau ferromagnétique; leur coupure basse fréquence est déterminée par la courbe I. Nous les appellerons «sondes à écran total» (SET).

- Celles du deuxième type sont les «transformateurs d'intensité ». Ils comportent un noyau ferromagnétique qui abaisse considérablement la limite basse fréquence définie alors par la courbe II. Mais ce noyau abaisse également la coupure haute fréquence comme nous le verrons ultérieurement.

Notons que, pour déterminer les coupures basse fréquence des deux types de sondes, nous avons supposé jusque-là que la résistance de mesure $R_{\mathrm{m}}$ placée entre les points A et B était nulle. En fait, il faut que le signal de mesure soit supérieur à $U_{\mathrm{m}}$ donc que :

$$
R_{\mathrm{m}} \mathrm{i}=R_{\mathrm{m}} \frac{I}{N} \geqslant U_{\mathrm{m}} .
$$

La présence de cette résistance $R_{\mathrm{m}}$ augmente la pénétration du champ dans la sonde; tout se passe comme si la résistivité $\rho$ devenait $\rho\left(1+\left(R_{\mathrm{m}} / \mathcal{R}\right)\right)$ où $\mathcal{R}=\rho(2 \pi r N / s)$ est la résistance de la sonde. Or, lorsque $R_{\mathrm{m}}=0$, la limitation inférieure en fréquence est donnée, d'après la figure 5 , par la relation

$$
\frac{e r_{0}}{\delta^{2}} \geqslant \frac{1}{\mu_{\mathrm{r}} g} \text { ou encore } \mu_{0} \frac{e r_{0}}{2} \cdot \frac{\omega}{\rho} \geqslant \frac{1}{\mu_{\mathrm{r}} g} .
$$

Lorsque $R_{\mathrm{m}}>0$, elle s'écrit donc (avec $r_{0} \simeq r$ )

$$
\mu_{0} \frac{e r}{2} \cdot \frac{\omega}{\rho\left(1+\frac{R_{\mathrm{m}}}{\mathcal{R}}\right)} \geqslant \frac{1}{\mu_{\mathrm{r}} g} .
$$

Comme les sondes sont de résistance très faible, nous avons $\mathcal{R} \ll R_{\mathrm{m}}$. Alors en remplaçant $e$ et $\mathcal{R}$

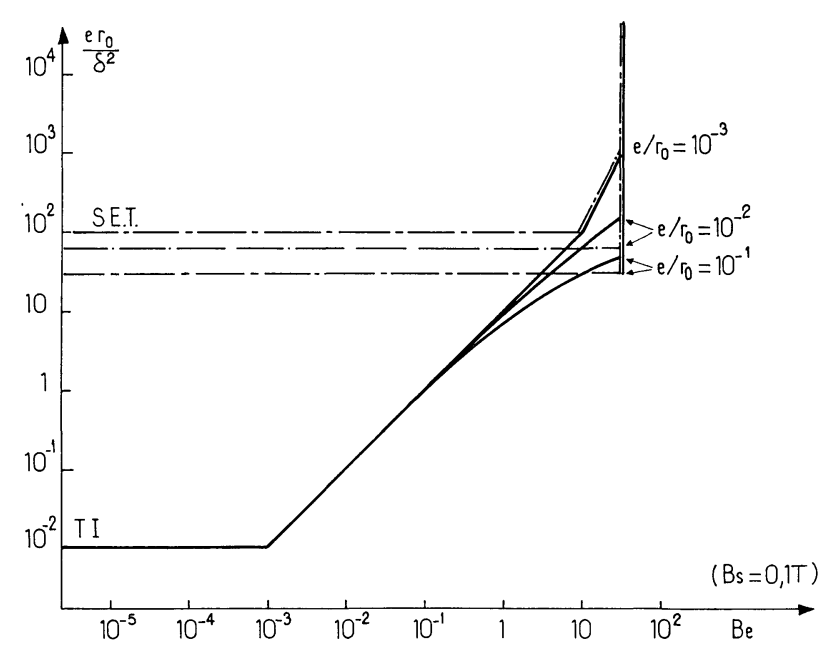

FIG. 5.

par leur valeur dans l'inéquation ci-dessus et en tenant compte de (16), nous obtenons la double inéquation :

$$
\mu_{0} \mu_{\mathrm{r}} g \frac{r^{2}}{2 R} N^{2} \omega \geqslant R_{\mathrm{m}} \geqslant \frac{U_{\mathrm{m}} \cdot N}{I} .
$$

L'existence d'une valeur $R_{\mathrm{m}}$ satisfaisant aux conditions imposées à la mesure est assurée si

$$
\mu_{0} \mu_{\mathrm{r}} g \frac{r^{2}}{2 R} I N \omega \geqslant U_{\mathrm{m}}
$$

ou encore : $10^{2} \cdot \pi N r^{2} \omega B_{\mathrm{e}} \geqslant U_{\mathrm{m}}\left(\mu_{\mathrm{r}} g=10^{2}\right)$. Cette limitation est analogue à celle que nous avons obtenue pour la CR (cf. (7a)), mais la fréquence limite est inférieure d'un coefficient $\mu_{\mathrm{r}} g=10^{2}$.

Nous remarquons que si la sonde et la résistance $R_{\mathrm{m}}$ sont données, le domaine local de mesure est délimité par deux conditions précises :

$$
\begin{aligned}
& \omega \geqslant \frac{2 R R_{\mathrm{m}}}{\mu_{0} \mu_{\mathrm{r}} g r^{2} N^{2}} \\
& I \geqslant \frac{U_{\mathrm{m}} N}{R_{\mathrm{m}}} .
\end{aligned}
$$

Les demi-droites $R_{\mathrm{m}} x$ et $R_{\mathrm{m}} y$ de la figure 6 représentent ces limitations.

ß) Echauffement. - En régime permanent, le courant maximum à mesurer dépendrait des moyens de refroidissement continu. Nous n'aborderons pas ce problème.

Nous ne considérerons ici que le cas de monoimpulsions de courant, les courants très intenses étant fournis seulement sous cette forme. Alors, la chaleur produite ne diffuse pas et est absorbée par élévation de température de la masse du conducteur. Pour simuler cette mono-impulsion, nous calculerons l'élévation de température $\Delta T$ pour un courant de pulsation $\omega$ durant une demi-période. Comme le signal n'est pas influencé sensiblement par la valeur 


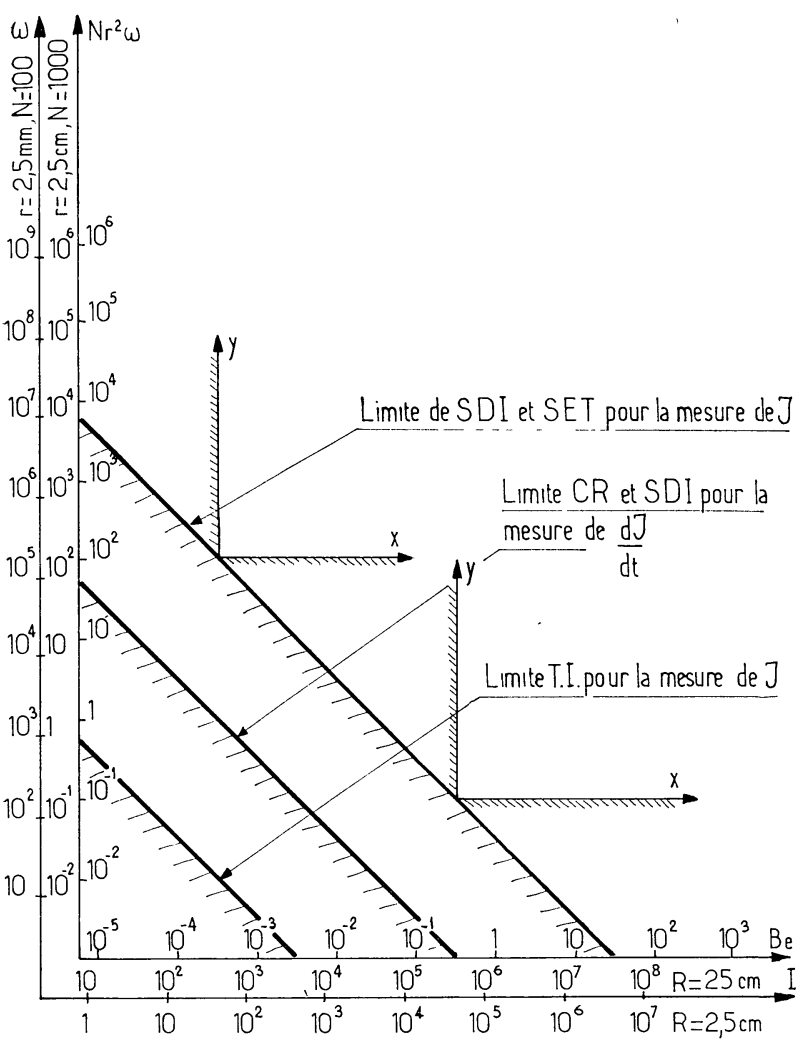

FIG. 6.

de la résistivité $\rho$ il n'est pas nécessaire d'imposer que celle-ci reste constante. Nous imposerons simplement que $\Delta T$ reste inférieur à $200^{\circ} \mathrm{C}$ pour que les isolants ne soient pas détériorés.

Deux cas se présentent suivant que $e<\delta$ ou $e>\delta$.

Si $e<\delta$, le courant est uniformément réparti dans le conducteur. L'induction $B_{\mathrm{e}}$ est liée à la densité de courant $k$ par la relation $B_{\mathrm{e}}=\mu_{0} k e$. Si le courant circule durant une demi-période $\pi / \omega$, l'élévation $\Delta T$ de température superficielle est alors telle que :

$$
\bar{\omega} c \Delta T=\frac{1}{2} \rho k^{2} \frac{\pi}{\omega}=\frac{\pi}{4 \mu_{0}} B_{\mathrm{e}}^{2} \cdot \frac{\delta^{2}}{e^{2}}
$$

( $\bar{\omega}$ et $c$ sont respectivement la masse spécifique et la capacité calorifique du conducteur).

Si $e \geqslant \delta$, le courant peut être considéré comme réparti dans la seule épaisseur de peau $\delta$. On obtient :

$$
\bar{\omega} c \Delta T=\frac{\pi}{4 \mu_{0}} B_{\mathrm{e}}^{2} .
$$

Nous retrouvons le résultat bien connu selon lequel l'élévation superficielle de température d'un conducteur massif soumis à une mono-impulsion de courant est proportionnelle au carré du champ et indépendante du terme d'impulsion. Dans le cas du cuivre, la condition $\Delta T \leqslant 200^{\circ} \mathrm{C}$ donne pour $B_{\mathrm{e}}$ la valeur limite :

$$
\left(B_{\mathrm{e}}\right)_{\mathrm{M}}=34 \mathrm{~T}
$$

Dans le cas où le courant circule en peau, ces sondes possèdent une limite supérieure, très élevée il est vrai, des champs mesurables.

Dans le cas où $e<\delta$, cette limite se calcule à partir de (18). En tenant compte de l'expression de $\left(B_{\mathrm{e}}\right)_{\mathrm{M}}$, on voit facilement que le champ $B_{\mathrm{e}}$ doit vérifier:

$$
B_{\mathrm{e}} \leqslant\left(B_{\mathrm{e}}\right)_{\mathrm{M}} \cdot \frac{e}{\delta}
$$

$B_{\mathrm{e}}$ est donc inférieur à $\left(B_{\mathrm{e}}\right)_{\mathrm{M}}$. Cette limitation par l'échauffement, qui fait intervenir le rapport $e / \delta$, sera exploitée dans la conclusion et portée sur la figure 5 .

$\gamma$ ) Limitation aux hautes fréquences. - Cette limitation est due à la présence de capacités parasites diverses et est de même origine pour les sondes à écran total et les transformateurs d'intensité d'une part et les sondes à débit interne d'autre part. Ce problème sera traité globalement dans le deuxième article.

3. Conclusion. - Nous venons d'étudier les principales limitations des sondes à écran total et des transformateurs d'intensité, excepté en ce qui concerne les limitations haute fréquence qui seront étudiées dans le deuxième article. L'ensemble des résultats obtenus est représenté sur le diagramme de la figure 5, où nous avons porté $e r_{0} / \delta^{2}$ en fonction de $B_{\mathrm{e}}$ pour diverses valeurs $\mathrm{du}$ rapport $e / r_{0}$. Pour une sonde donnée, ce diagramme représente $\omega$ en fonction de $I$. Pour le tracer, nous avons admis $B_{\mathrm{S}}=0,1 \mathrm{~T}$ comme l'indique Fouillé [4]. Il sera complété dans le prochain article par l'étude des limitations haute fréquence et discuté à ce moment-là.

IV. Sondes à débit interne [5]. - 1. Signaux. La structure d'une SDI se rapproche beaucoup de celle d'une $\mathrm{CR}$ mais son fonctionnement en diffère notablement puisqu'elle est traversée par un courant $i$ assez important et que l'impédance de la boîte de mesure est très faible. D'autre part, sa résistance $\mathcal{R}=N \rho(2 \pi r / s)$ est très élevée contrairement à celle d'une sonde à écran total.

Si $L=\mu_{0} N^{2}\left(r^{2} / 2 R\right)$ est la self de la sonde (la self du fil de retour $A^{\prime} B$ est négligeable dès que ce fil est assez gros) et si nous admettons que la mesure n'apporte aucune perturbation, le courant $i$ est déterminé par l'équation:

$$
\begin{aligned}
\mathcal{R} i+L \frac{\mathrm{d} i}{\mathrm{~d} t} & =-N \frac{\mathrm{d}\left(\pi r^{2} \mathcal{B}_{\mathrm{e}}\right)}{\mathrm{d} t} \\
& =-\mu_{0} \frac{r^{2}}{2 R} \cdot N \frac{\mathrm{d} J}{\mathrm{~d} t}=-\frac{L}{N} \cdot \frac{\mathrm{d} J}{\mathrm{~d} t} .
\end{aligned}
$$

Pour une pulsation $\omega$ donnée, on peut écrire

$$
\overline{\mathrm{i}}=-\frac{1}{N} \cdot \frac{1}{\frac{\mathcal{R}}{j L \omega}+1} \cdot \bar{I} .
$$

Si $L \omega / \mathcal{R} \ll 1,(20)$ devient $i \approx-L / \mathcal{R} N \mathrm{~d} \mathfrak{J} / \mathrm{d} t$ le courant $i$ est proportionnel à $\mathrm{d} J / \mathrm{d} t$; on peut donc 
atteindre cette grandeur $i$ en prenant comme impédance de mesure une résistance et la grandeur $\mathfrak{J}$ en intégrant $\mathrm{d} J / \mathrm{d} t$ à l'aide d'une capacité.

De manière plus générale, si la condition $L \omega / \mathcal{R} \ll 1$ n'est plus strictement vérifiée, on peut mesurer le courant $i$ ou les expressions qui lui sont attachées en plaçant entre $\mathrm{A}$ et $\mathrm{B}$ une impédance $\bar{Z}$. La tension $U$ qui apparaît aux bornes de cette impédance constitue alors le signal de mesure proprement dit. Pour la partie du courant ayant la pulsation $\omega$, cette tension s'obtient immédiatement. On a :

$$
-\bar{U}=\frac{1}{N} \frac{j L \omega \bar{Z}}{\mathcal{R}+j L \omega+\bar{Z}} \bar{I} .
$$

Si l'on désire mesurer $\mathrm{d} J / \mathrm{d} t$, il faut que $\bar{U}$ soit proportionnel à $j \omega \bar{I}$, donc que :

$$
\bar{U}=\bar{U}_{\mathrm{R}}=-\chi_{\mathrm{R}} j \omega \bar{I},
$$

où $\chi_{R}$ est la constante de proportionnalité de la mesure. L'impédance de mesure $\bar{Z}_{\mathrm{R}}$ est alors donnée par :

$$
\bar{Z}_{\mathrm{R}}=\frac{N \chi_{\mathrm{R}}}{L-N \chi_{\mathrm{R}}}(\mathcal{R}+j L \omega) .
$$

Elle se réalise facilement par la mise en série d'une résistance $R_{\mathrm{m}}$ de valeur

$$
R_{\mathrm{m}}=\frac{N \chi_{\mathrm{R}}}{L-N \chi_{\mathrm{R}}} \mathcal{R}
$$

et d'une inductance de correction $l_{\mathrm{m}}$ égale à $N \chi_{\mathrm{R}} /\left(L-N \chi_{\mathrm{R}} L\right)$, donc telle que, $l_{\mathrm{m}} / R_{\mathrm{m}}=L / \mathcal{R}$. Il faut, bien sûr, que $\chi_{\mathrm{r}}$ soit tel que $\chi_{\mathrm{R}} \leqslant L / N$. La proportionnalité entre $\mathcal{U}_{\mathrm{R}}$ et $\mathrm{d} J / \mathrm{d} t$ est alors assurée indépendamment de la fréquence. On a :

$$
-\bar{U}_{\mathrm{R}}=\frac{L R_{\mathrm{m}}}{\mathcal{R}+R_{\mathrm{m}}} j \omega \bar{I} .
$$

Si l'on désire maintenant mesurer le courant $\mathfrak{J}$ proprement dit, on doit avoir :

$$
\bar{U}=\bar{U}_{\mathrm{D}}=-\chi_{\mathrm{c}} \bar{I}
$$

où $\chi_{\mathrm{c}}$ est la constante de proportionnalité de la mesure. Pour satisfaire à l'égalité (25), il faut placer entre les points $\mathrm{A}$ et $\mathrm{B}$ une impédance $Z_{\mathrm{D}}$ donnée par l'expression :

$$
Z_{\mathrm{D}}=\chi_{\mathrm{c}} N \frac{\Re+j L \omega}{j L \omega-\chi_{\mathrm{c}} N} .
$$

Malheureusement, cette impédance n'est pas réalisable par un montage capacité-self-résistance passif et nous ne pourrons satisfaire à l'égalité (25) que de manière approchée. Nous choisirons de placer entre $A$ et $\mathrm{B}$ une impédance $\bar{Z}_{\mathrm{c}}$ telle que :

$$
\bar{Z}_{\mathrm{c}}=\chi_{\mathrm{c}} N\left[\frac{\mathcal{R}}{j L \omega}+1\right]
$$

constituée par la mise en série d'une capacité

$$
C_{\mathrm{m}}=\frac{L}{\mathcal{R} \chi_{\mathrm{c}} N}
$$

et d'une résistance de correction $r_{\mathrm{m}}=\chi_{\mathrm{c}} N$, soit telle que $r_{\mathrm{m}} C_{\mathrm{m}}=L / \mathcal{R}$. La relation liant la mesure $\bar{U}_{\mathrm{c}}$ et $\bar{I}$ devient :

$$
-\bar{U}_{\mathrm{c}}=\frac{\chi_{\mathrm{c}}}{1+\frac{\chi_{\mathrm{c}} N}{j L \omega}} \bar{I}=\frac{L}{N \mathcal{R} C_{\mathrm{m}}}\left[\frac{1}{1+\frac{1}{j \mathcal{R} C_{\mathrm{m}} \omega}}\right] \bar{I} .
$$

La proportionnalité entre la mesure $\mathcal{U}_{c}$ et le courant $J$ n'est pas toujours assurée. Pour que l'erreur soit inférieure à $g$ il faut que :

$$
R C_{\mathrm{m}} \omega \geqslant \frac{1}{g} \gg 1 \text {. }
$$

L'erreur introduite par le facteur $1 / j \mathfrak{R} C_{\mathrm{m}} \omega$ correspond essentiellement à un déphasage du signal ; elle croît lorsque la fréquence baisse. La condition (29) est la condition classique permettant l'intégration d'un signal par une capacité.

Bien évidemment, $U_{\mathrm{c}}$ et $U_{\mathrm{R}}$ ne resteront proportionnels à $\mathfrak{J}$ et $\mathrm{d} J / d t$ que si la résistance $\mathcal{R}$ est invariante au cours du temps. Pour cela, le fil du bobinage doit avoir un diamètre inférieur à l'épaisseur de peau et la résistivité doit rester invariante.

2. Domaine D’utilisation. - Nous ne considérerons ici que les limitations apportées par l'élévation de température $\Delta T$ et les conditions sur les tensions de mesure. L'influence des capacités parasites sera étudiée de façon globale dans le deuxième article.

๙) Elévation de température. - L'élévation de température devant rester faible, nous admettrons pour la calculer que $\rho$ est une constante. Alors :

$$
\bar{\omega} c \Delta T=\rho \int \frac{i^{2}}{s^{2}} \mathrm{~d} t .
$$

Considérons, comme pour les TI et les SET, une impulsion de courant qui dure une demi-période $\pi / 2 \omega$. En supposant $L / \mathcal{R} \ll 1, i$ est donné par $\left(20^{\prime}\right)$ et vaut :

$$
i=-\frac{L}{\mathcal{R} N} \frac{\mathrm{d} J}{\mathrm{~d} t}=\frac{L}{\mathcal{R} N} \omega I \sin \omega t
$$

$\Delta T$ est donc déterminé par :

$$
\bar{\omega} c \Delta T=\rho \int_{0}^{\pi / 2 \omega}\left(\frac{L}{\mathcal{R} N} \omega I\right)^{2} \sin ^{2} \omega t \mathrm{~d} t
$$

ce qui donne, en remplaçant $L$ et $\mathcal{R}$ par leurs valeurs et en exprimant $I$ en fonction de $B_{\mathrm{e}}$ :

$$
\Delta T=\frac{\pi}{8} \cdot \frac{1}{\rho \bar{\omega} c} \cdot r^{2} \omega B_{\mathrm{e}}^{2} .
$$

L'élévation de température doit être suffisamment faible pour que les isolants ne soient pas détériorés et que la résistance $\mathcal{R}$ de la sonde soit pratiquement invariante. De plus, le rapport $R / L \omega$ ne doit pas 
être petit. On est donc amené à bobiner la sonde avec un matériau de grande résistivité peu sensible aux variations de température. Typiquement, nous prendrons un alliage nickel-chrome pour lequel $\rho \sim 1,3 \times 10^{-6} \Omega$.m et dont le coefficient de température vaut $2 \times 10^{-5} /{ }^{\circ} \mathrm{K}$ et nous admettrons pour $\Delta T$ une limite de $200^{\circ}$. En introduisant les paramètres physiques du matériau, on obtient l'inéquation numérique :

$$
r^{2} \omega \leqslant \frac{5,4 \times 10^{2}}{B_{\mathrm{e}}^{2}} .
$$

Si le courant n'est pas formé d'une seule impulsion mais de $k$ demi-périodes, la condition devient bien sûr $r^{2} \omega \leqslant 5,4 \times 10^{2} / k B_{\mathrm{e}}^{2}$. Cependant s'il y a diffusion de la chaleur et si la sonde est refroidie, la condition précédente peut être modifiée de façon appréciable.

ß) Grandeur des signaux. - Pour que les signaux $\bar{U}_{\mathrm{R}}$ et $\bar{U}_{\mathrm{c}}$ soient convenables, leurs amplitudes respectives $U_{\mathrm{R}}$ et $U_{\mathrm{c}}$ doivent être supérieures à la valeur minimale $U_{\mathrm{m}}$. Par ailleurs, la condition (29) doit être vérifiée pour que $\mathcal{U}_{c}$ reste proportionnel à $\mathfrak{J}\left(\mathcal{U}_{\mathrm{R}}\right.$ étant proportionnel à $\mathrm{d} J / \mathrm{d} t$ quelle que soit la fréquence).

En ce qui concerne $U_{\mathrm{R}}$, nous devons donc avoir :

$$
U_{\mathrm{R}}=\chi_{\mathrm{R}} \omega I \geqslant U_{\mathrm{m}} \text {. }
$$

Or, la valeur maximale de $U_{\mathrm{R}}$ est atteinte pour la valeur maximale $L / N$ de $\chi_{\mathrm{R}}$. Alors, $U_{\mathrm{R}}=L \omega I / N$, $Z_{\mathrm{R}}$ est infini et la SDI est devenue une sonde sans débit, c'est-à-dire une CR. En particulier, les limitations aux basses fréquences sont les mêmes pour les SDI et les CR.

En ce qui concerne $U_{\mathrm{c}}$, nous devons satisfaire la condition (29) :

$$
\mathcal{R} C_{\mathrm{m}} \omega \geqslant \frac{1}{g}
$$

et la condition à l'amplitude du signal :

$$
U_{\mathrm{c}}=\frac{L}{N \mathcal{R} C_{\mathrm{m}}} I \geqslant U_{\mathrm{m}},
$$

l'ensemble de ces deux inéquations pouvant s'écrire :

$$
\frac{U_{\mathrm{m}}}{I} \leqslant \frac{L}{N \Re C_{\mathrm{m}}} \leqslant g \frac{L \omega}{N} .
$$

L'existence d'une valeur de la capacité $C_{\mathrm{m}}$ satisfaisant aux conditions ci-dessus est assurée lorsque :

$$
U_{\mathrm{m}} \leqslant g \frac{L \omega I}{N}
$$

soit lorsque :

$$
U_{\mathrm{m}} \leqslant \pi g N r^{2} \omega B_{\mathrm{e}} .
$$

Cette condition (32) définit le domaine d'utilisation aux basses fréquences des SDI lorsqu'on s'intéresse à la mesure du courant. Elle est portée sur le diagramme de la figure 6 pour les valeurs habituelles $U_{\mathrm{m}}=10^{-3} \mathrm{~V}$ et $g=10^{-2}$.

3. Conclusion. - Nous venons tout d'abord de préciser les impédances de mesure que l'on doit mettre aux bornes d'une SDI. Pour mesurer $J$, il faut une capacité $C_{\mathrm{m}}$ à laquelle on adjoint une résistance de correction, $r_{\mathrm{m}}$; pour mesurer $\mathrm{d} J / \mathrm{d} t$ il faut une résistance $R_{\mathrm{m}}$ à laquelle on adjoint une self de correction $l_{\mathrm{m}}$. La présence des impédances de correction $r_{\mathrm{m}}$ et $l_{\mathrm{m}}$ permet d'utiliser ces sondes non seulement lorsque $L \omega / \mathcal{R} \ll 1$ mais aussi lorsque $L \omega / \mathcal{R} \simeq 1$.

Nous avons par ailleurs délimité le domaine d'utilisation de ces sondes en ne nous préoccupant pas de l'existence de capacités parasites. Les résultats concernant la limitation basse fréquence sont portés sur le diagramme de la figure 6, à la fois pour les SDI et pour tous les autres types de sondes. Én ce qui concerne la mesure de $\mathrm{d} J / \mathrm{d} t$, les $\mathrm{CR}$ et les SDI sont équivalentes. La limitation relative à la mesure de $\mathfrak{J}$ est la même pour les SDI et les SET ; elle est $\mu_{\mathrm{r}}$ fois, soit $10^{4}$ à $10^{5}$ plus basse pour les TI qui seront donc mieux adaptés aux mesures basse fréquence. Remarquons que, lorsqu'on mesure $\mathfrak{J}$ avec une SDI, une SET ou un TI, le domaine d'utilisation est en fait défini par deux demi-droites $\mathrm{O}^{\prime} x$ et $\mathrm{O}^{\prime} y$ lorsque la sonde et l'impédance de mesure sont données; nous avons tracé sur la figure 6 les demi-droites correspondant à une SET donnée avec sa résistance de mesure $R_{\mathrm{m}}$ d'une part et à une SDI donnée avec sa capacité de mesure $C_{\mathrm{m}}$, d'autre part.

V. Conclusions générales. - Nous venons de définir les domaines d'utilisation des sondes inductives lorsque le champ extérieur possède une complète symétrie de révolution. Excepté pour les échauffements, ces limitations concernent essentiellement les basses fréquences. Les diagrammes 2, mais surtout 5 et 6 résument ces résultats. Les limitations haute fréquence dues aux anisotropies des distributions angulaires seront étudiées dans le deuxième article qui nous permettra de tracer le diagramme complet d'utilisation de ces sondes.

\section{Bibliographie}

[1] PARK (J. H.), Journal of Research of the National Bureau of Standards, 1947, 39, 191.

[2] Rogowski (W.) et Steinhaus (W.), Arch. Elektrotech., $1912,1,141$

[3] BRuhat (G.), «Electricité » remaniée par Goudet (G.), Masson, éd., Paris, 1959.
[4] FouILLÉ (A.), Electrotechnique à l'usage des Ingénieurs, Dunod, éd., Paris, 1969, Tome I.

[5] Ces sondes font l'objet du brevet « ANVAR » $\mathrm{n}^{\circ} 72.01$. 226. 